\section{Causative Pathogens, Antibiotic Susceptibility, and Characteristics of Patients with Bacterial Septic Arthritis over Time}

To the Editor:

Septic arthritis is an important concern for rheumatologists in the evaluation of joint disease. The incidence of these infections is estimated at 4-10 cases per 100,000 patient-years, and the mortality rate ranges between $4 \%$ and $50 \%{ }^{1,2}$. Management of septic arthritis remains a challenge because inappropriate treatment can lead to irreversible joint destruction. Over the past decade, the prevalence of antimicrobial-resistant pathogens, including methicillin-resistant Staphylococcus aureus (MRSA) and multidrug-resistant (MDR) gram-negative bacilli (GNB), has increased in the United States. More recently, S. aureus with reduced susceptibility to vancomycin has been reported $^{3,4}$. There are only limited data that describe the incidence and outcomes of patients with septic arthritis caused by S.aureus with reduced susceptibility to vancomycin or MDR GNB. The objective of our study was to investigate the trends of causative pathogens, antibiotic susceptibility, and characteristics of patients with bacterial septic arthritis in the era of multidrug resistance. The institutional review board of Boston Medical Center approved this analysis (H-34459).

A retrospective chart review of all incident inpatient cases of septic arthritis with positive synovial cultures from January 2000 to September 2015 was conducted at Boston Medical Center, a tertiary medical center. We reviewed patient charts and included the cases defined by the following: joint fluid cellular profile compatible with infection and not explained by an underlying rheumatologic disorder. MDR GNB was defined as resistance to at least 1 agent in 3 or more classes of antibiotics ${ }^{5}$.

Patient demographics, clinical information, and antibiotic resistance patterns were compared. Because there have been reports of decreasing susceptibility to vancomycin in MRSA since $2005^{3}$, we separated calendar time into an early period (2000-2004) and a later period (2005-2015). The proportion of septic arthritis cases in the 2 periods was compared using the chi-squared test. We also compared clinical outcomes between the 2 periods using Mann-Whitney U test and chi-square tests. Clinical outcomes included median hospital length of stay (LOS), intensive care unit (ICU) treatment, and in-hospital mortality. Mortality was defined as death occurring during the same hospital stay. Analyses were performed with SAS software version 9.3.

There were 128 cases of septic arthritis that met the inclusion criteria; 40 in the period 2000-2004 and 88 in the period 2005-2015. Native joint infection accounted for $72 \%$ (92 of 128 cases). Baseline demographic and characteristics of the patients are shown in Table 1. Staphylococcal infections accounted for $73 \%$ (93 of 128 cases) of all cases of septic arthritis. MRSA accounted for $22 \%$ ( 20 of 92 cases) of native joint infection and $11 \%$ ( 4 of 36 cases) of prosthetic joint infection. MRSA septic arthritis cases did not differ significantly between the first and second period (15\% vs $20 \%$ of cases, $p=0.46$ ). There was 1 case of MRSA septic arthritis (native joint infection) with a vancomycin minimum inhibitory concentration $\geq 1.5 \mu \mathrm{g} / \mathrm{ml}$ in the first period, and 2 cases ( 1 native joint and 1 prosthetic joint infection) in the second period. There was 1 case (native joint infection) of MDR GNB (Escherichia coli) in the second period.

The outcomes are shown in Table 2. The incidence of concomitant endocarditis or sepsis was similar between patients with methicillin-sensitive S. aureus (MSSA) and MRSA (9.7\% vs $8.3 \%$ and $51.7 \%$ vs $56.5 \%$, respectively). Median LOS was similar between patients with MSSA and MRSA (11 days vs 14 days, $p=0.59$ ). In-hospital mortality rate in cases of MSSA and MRSA septic arthritis was $6.7 \%$ and $8.3 \%$, respectively. Of the 3 cases of MRSA septic arthritis with reduced vancomycin susceptibility, 2 developed sepsis requiring ICU admission. LOS was 9, 19, and 30 days for the 3 cases and none died during hospitalization. One case of MDR GNB (E. coli) developed severe sepsis that required ICU admission.

In this single-center analysis, there were few cases of septic arthritis caused by reduced vancomycin susceptibility or MDR GNB organisms. Overall, the distribution and sensitivity of bacteria causing septic arthritis,
Table 1. Baseline demographic and clinical characteristics of 128 patients with septic arthritis. Data are n (\%) unless otherwise specified.

\begin{tabular}{lcc}
\hline Characteristics & $\begin{array}{c}2000-2004, \\
\mathrm{n}=40\end{array}$ & $\begin{array}{c}2005-2015, \\
\mathrm{n}=88\end{array}$ \\
\hline Age, yrs, median (IQR) & $56(47-66)$ & $56(47-65)$ \\
Male & $31(77.5)$ & $65(73.9)$ \\
Intravenous drug use & $7(18.4)$ & $21(23.9)$ \\
Diabetes mellitus & $13(33.3)$ & $27(30.7)$ \\
Immunosuppressive agent & $4(10.3)$ & $7(8.0)$ \\
Malignancy & $3(7.7)$ & $9(10.2)$ \\
HIV & $2(5.1)$ & $5(5.7)$ \\
AIDS & $1(2.6)$ & $2(2.3)$ \\
Hepatitis C & $13(33.3)$ & $33(37.5)$ \\
Cirrhosis & $2(5.1)$ & $5(5.7)$ \\
Endstage renal disease & $9(23.1)$ & $7(8.0)$ \\
Rheumatoid arthritis & $0(0)$ & $1(1.1)$ \\
Presence of skin infection & $6(15.8)$ & $26(29.6)$ \\
Prosthetic infection & $8(20.0)$ & $28(31.8)$ \\
WBC, thousands, median (IQR) & $12.2(8.9-16.2)$ & $10.3(7.2-15.6)$ \\
ESR, median (IQR) & $81(62-101)$ & $73(45-99)$ \\
Synovial fluid cell count, thousands, & & \\
$\quad$ median (IQR) & $64.5(29.2-112.0)$ & $73.4(33.4-120.0)$ \\
Poly, \% (IQR) & $93(89-95)$ & $93(89-95)$ \\
Positive blood culture & $18(48.7)$ & $35(40.2)$ \\
\hline
\end{tabular}

* Immunosuppressive agent included prednisone, tumor necrosis factor inhibitor, or chemotherapy within 3 months. IQR: interquartile range; HIV: human immunodeficiency virus; WBC: white blood cells; ESR: erythrocyte sedimentation rate; poly: polymorphonuclear neutrophils.

including MRSA and MDR GNB, did not differ between the 2 study periods (2000-2004 vs 2005-2015), although power was limited owing to small numbers. Two of the 3 cases of MRSA septic arthritis with reduced vancomycin susceptibility occurred in the second period (2005-2015). Two cases developed sepsis, requiring ICU admission.

The present study had several limitations. First, because it was a single-center study, these results may not be generalizable to a different patient population. Second, there were only 3 cases of MRSA septic arthritis with reduced vancomycin susceptibility and 1 case of MDR GNB septic arthritis. Although the septic arthritis cases caused by these organisms were associated with worse outcomes, we were unable to draw definitive conclusions.

The distribution and sensitivity of bacteria causing septic arthritis did not differ between the 2 study periods. Empiric coverage with drugs active against $S$. aureus with reduced susceptibility to vancomycin and for MDR GNB is not warranted at current prevalence levels.

SADAO JINNO, MD, MSc, Section of Rheumatology, Department of Medicine, Boston University School of Medicine; CAROL A. SULIS, MD, Section of Infectious Diseases, Department of Medicine, Boston University School of Medicine; MAUREEN D. DUBREUIL, MD, MSc, Section of Rheumatology, and Section of Clinical Epidemiology Research and Training Unit, Department of Medicine, Boston University School of Medicine, and VA Boston Healthcare System, Boston, Massachusetts, USA. Address correspondence to Dr. S. Jinno, Section of Rheumatology, Department of Medicine, Boston University School of Medicine, Boston, Massachusetts 02118, USA. E-mail: sadaoj@gmail.com

\section{REFERENCES}

1. Fangtham M, Baer AN. Methicillin-resistant Staphylococcus aureus arthritis in adults: case report and review of the literature. Semin Arthritis Rheum 2012;41:604-10. 
Table 2. Outcomes of septic arthritis across different organisms. Data are n (\%) unless otherwise indicated.

\begin{tabular}{lccccc}
\hline Variables & $\begin{array}{c}\text { MSSA, } \\
\mathrm{n}=60\end{array}$ & $\begin{array}{c}\text { MRSA, } \\
\mathrm{n}=24\end{array}$ & $\begin{array}{c}\text { CoNS, } \\
\mathrm{n}=7\end{array}$ & $\begin{array}{c}\text { Streptococci, } \\
\mathrm{n}=22\end{array}$ & $\begin{array}{c}\text { GNB, } \\
\mathrm{n}=10\end{array}$ \\
\hline Concomitant endocarditis & $6(9.7)$ & $2(8.3)$ & 0 & 0 & 0 \\
Sepsis* & $30(51.7)$ & $13(56.5)$ & 0 & $5(22.7)$ & 0 \\
Surgical intervention & $48(81.4)$ & $19(82.6)$ & $7(100)$ & $5(68.2)$ & $10(100)$ \\
ICU stay, days & $15(25.4)$ & $7(29.1)$ & $1(14.3)$ & $5(22.7)$ & $1(10.0)$ \\
LOS, days, median (IQR) & $11(8-16)$ & $14(7-19)$ & $9(8-11)$ & $9(5-14)$ & $7(7-12)$ \\
Death & $4(6.7)$ & $2(8.3)$ & 0 & 0 & 0 \\
\hline
\end{tabular}

* Two or more systemic inflammatory response syndrome criteria. MSSA: methicillin-sensitive Staphylococcus aureus; MRSA: methicillin-resistant S. aureus; CoNS: coagulase negative staphylococci; GNB: gram-negative bacilli; ICU: intensive care unit; LOS: length of (hospital) stay; IQR: interquartile range.

2. Ferrand J, El Samad Y, Brunschweiler B, Grados F, Dehamchia-Rehailia N, Sejourne A, et al. Morbimortality in adult patients with septic arthritis: a three-year hospital-based study. BMC Infect Dis 2016;16:239.

3. Wang G, Hindler JF, Ward KW, Bruckner DA. Increased vancomycin MICs for Staphylococcus aureus clinical isolates from a university hospital during a 5-year period. J Clin Microbiol 2006;44:3883-6.

4. Rybak MJ, Leonard SN, Rossi KL, Cheung CM, Sader HS, Jones $\mathrm{RN}$. Characterization of vancomycin-heteroresistant Staphylococcus aureus from the metropolitan area of Detroit, Michigan, over a 22year period (1986 to 2007). J Clin Microbiol 2008;46:2950-4.

5. Magiorakos A-P, Srinivasan A, Carey RB, Carmeli Y, Falagas ME, Giske CG, et al. Multidrug-resistant, extensively drug-resistant and pandrug-resistant bacteria: an international expert proposal for interim standard definitions for acquired resistance. Clin Microbiol Infect 2012;18:268-81.

J Rheumatol 2018;45:5; doi:10.3899/jrheum.171115 